\title{
A combined statistical and reactive transport modeling approach to demystifying the terrestrial aquatic interface
}

\author{
BHAVNA ARORA ${ }^{1}$, HANNAH WATERHOUSE ${ }^{2}$, SWATHI \\ VENKATESH $^{3}$, DIPANKAR DWIVEDI ${ }^{1}$, PETER S NICO $^{1}$ \\ AND MARGARET ZIMMER ${ }^{4}$ \\ ${ }^{1}$ Lawrence Berkeley National Laboratory \\ ${ }^{2}$ University of California, Berkeley \\ ${ }^{3}$ Amrita Vishwa Vidyapeetham \\ ${ }^{4}$ University of California, Santa Cruz \\ Presenting Author: barora@lbl.gov
}

Although biogeochemical hot moments are known to account for a high percentage of nutrient cycling within terrestrial and aquatic ecosystems, the ability to effectively identify hot moments and their associated controls remains a significant challenge. Using a combination of hydrological, solid and aqueous geochemical, geological, microbial and meteorological datasets, we seek to identify relevant hot moments and quantify which properties most explain the temporal variability in solute concentrations across a diversity of terrestrial aquatic interface (TAI) settings. Statistical approaches combined with reactive transport modeling offer the opportunity to systematically interrogate complex, multivariate datasets without making assumptions regarding the nature of temporal structure and dependencies implicit in these datasets. We develop and test these approaches at two sites: (1) a pristine mountainous watershed in East River, Colorado, and (2) a tidally-controlled estuarine environment in Central California. Statistical analysis results demonstrate that annual perturbations constitute hot moments that drive nitrogen cycling across sites; however, other frequencies (daily tidal, seasonal, bi-annual) dominate individual sites based on specific site characteristics. To further explore the dominant controls driving these elevated concentrations of nitrate, we use a reactive transport modeling approach to explore how the frequency and duration of ponding conditions impact nitrogen concentrations in a testbed approach with different stratigraphic configurations. Overall, our results suggest that lithologic heterogeneity, antecedent soil moisture conditions and depth to the water table are important controls on nitrate concentrations. 